\title{
3 Analytische Zugänge zur Gleichzeitigkeit multipler Aktivitäten im Rahmen der konversationsanalytischen Methodologie
}

Koordinative Verbünde aus aufeinander bezogenen, multimodal verfassten Beteiligungsweisen in mehreren simultan relevanten Handlungssequenzen stellen komplexe kommunikative Situationen dar. Um die interaktionalen Verfahren bei der Bearbeitung von gleichzeitiger Relevanz erschließen zu können, bedarf es eines analytischen Rahmens, der die sequentielle Organisiertheit der Handlungen rekonstruieren und die Gestalthaftigkeit multimodaler Simultanität fassen kann, ohne den Interagierenden Absichten und Einstellungen durch den ${ }^{\star}$ die Forscher^in zuzuschreiben. Da die Konversationsanalyse an der emischen Perspektive der Teilnehmenden in situ ansetzt, ist sie in besonderem Maße für dieses Vorhaben geeignet. Denn im Rahmen von konversationsanalytischen Arbeiten gehen Forschende davon aus, dass Interagierende in alltäglichen Interaktionen nicht in die Köpfe der anderen Beteiligten schauen können. Sie sind vielmehr darauf angewiesen, dass die anderen Teilnehmenden einander ihre Interpretationen der laufenden Interaktion als multimodale Gestalt im Rahmen des next turn proof procedure (Sacks, Schegloff \& Jefferson 1974) gegenseitig öffentlich anzeigen: „it obliges its participants to display to each other, in a turn's talk, their understanding of other turns' talk“ (Sacks, Schegloff \& Jefferson 1974: 728). Der analytische Zugriff auf soziale Interaktion erfolgt also im Rahmen der Konversationsanalyse über die Teilnehmendeninterpretationen anhand des next turn proof procedures.

Jedoch stellt besonders die multimodale Verfasstheit von Aktivitäten Analysierende vor mehrfache Herausforderungen. Zum einen lassen sich die anhand von verbalen Interaktionen entwickelten Konzepte (wie z. B. das der Teilnehmendeninterpretation zugrundeliegende Display-Konzept) nicht ohne Weiteres auf multimodale Interaktionen übertragen (vgl. Schmitt 2005) und zum anderen ist oft für Forschende nicht eindeutig $\mathrm{zu}$ bestimmen, welche multimodalen Informationen die Beteiligten relevant setzen (vgl. Deppermann 2013). Besonders herausfordernd wird der analytische Zugriff auf Interaktionen, wenn Teilnehmendeninterpretationen sprachfrei organisiert werden, was bei der Koordination von multiplen Aktivitäten beobachtet werden kann. Das Ziel dieses Kapitels ist es, die Grundannahmen und Grenzen des next turn proof procedures als analytischen Zugriff für verbal abstinente Beteiligungsweisen zu diskutieren (3.1) und mithilfe des Displayings und Monitorings als Koordinationsprozesse des interaktiven Vollzugs für multimodale Fragestellungen zu erweitern (3.2). 


\subsection{Die Grundlagen und Grenzen des next turn proof procedures}

Wenn Interagierende Interaktionsbeiträge produzieren, beziehen sie sich in der Regel auf vorherige Beiträge, weshalb jede interaktionale Handlung kontextabhängig und situiert ist (Sacks et al. 1974). Mit der Realisierung eines Beitrags innerhalb eines Redezugs (Turn) zeigen Interagierende ihre Interpretation der vorausgegangenen Turns an. Die Folge aufeinander bezogener Äußerungen, geregelt durch die Mechaniken des Sprecherwechsels (Sacks, Schegloff \& Jefferson 1974), nennen konversationsanalytisch Forschende Sequenzen (Heritage 2005). Als zentrale Analysemethode der Methodologie der Konversationsanalyse leistet die schrittweise Sequenzanalyse die Bedeutungsrekonstruktion in Interaktionen. Damit werden Interaktionen als Resultate geordneter und ordnender Praktiken der Teilnehmenden innerhalb der spezifischen Situation erkennbar. Die Ordnung sozialer Interaktion, deren Bestandteil multiple Aktivitäten sein können, steht dabei nicht vorher fest, sondern ist das Ergebnis der lokalen Aushandlung der Teilnehmenden im Vollzug als Vollzugswirklichkeit (,,a member‘s practical accomplishment“ Garfinkel 1967: 288). Gemäß der Maxime „order at all points” (Sacks 1984: 21) ist im Rahmen der Vollzugswirklichkeit kein (sprachliches) Zeichen per se überflüssig und kann für die Interaktionsorganisation relevant sein. Vielmehr sind alle Beteiligten gleichermaßen daran beteiligt, situativ gebundene und kontextabhängige Bedeutungsvorschläge für die anderen Interagierenden zu produzieren (Dausendschön-Gay \& Krafft 2002: 32-33). Diese Bedeutungsvorschläge werden von den Ko-Teilnehmenden in ihren jeweiligen Interaktionsbeiträgen angenommen oder abgelehnt und somit als ihre Interpretation der vorherigen Beiträge erkennbar. Was dabei erkennbar wird, bildet als sogenannte Teilnehmendeninterpretation die Grundlage des next turn proof procedures (Sacks, Schegloff \& Jefferson 1974) als zentrales Analyseverfahren der Konversationsanalyse. Demnach ergibt sich der analytische Zugang innerhalb der konversationsanalytischen Methodologie aus denjenigen Prinzipien, die auch bei der Konstitution von Interaktionen durch die Teilnehmenden Anwendung finden (Deppermann 2000: 97-98). Nur durch diesen datengeleiteten ${ }^{14}$ Ansatz vermag die Konversationsanalyse ihrem „rekonstruktiven Anspruch“ gerecht zu werden: „Es interessiert nicht, wie ein Analytiker Gesprächsaktivitäten aufgrund seiner Intuitionen oder theoretischen Ausrichtung versteht. Es geht vielmehr darum zu rekonstruieren, wie die

14 Daten meint hier die Gesamtheit einer audio(-visuellen) Aufnahme bzw. eine zu Analysezwecken gekürzte Sequenz - dabei wird jedoch immer darauf geachtet, dass die Interaktionen nicht aus ihrem Kontext gerissen werden, in dem sie hervorgebracht worden sind und nicht von scheinbar überflüssigen Elementen bereinigt werden. 
Gesprächsteilnehmenden selbst einander verstehen und an welchen Regeln oder Prinzipien sie sich dabei orientieren“ (Deppermann 2000: 98-99).

Diese gegenseitige Orientierung der Teilnehmenden setzt voraus, dass Interagierende ihre Interaktionsbeiträge füreinander so gestalten, dass diese Beiträge für andere erkennbar und darauf reagierbar sind. Bei Garfinkel (1967: xii) heißt diese Erkennbar-und-darauf-Reagierbarkeit ${ }^{15}$ accountable. Eine Handlung ist im ethnomethodologischen Sinne accountable, wenn andere Beteiligte sie als eine bestimmte Handlung verstehen können (Garfinkel 1967: 1) und sie dies in ihren Teilnehmendeninterpretationen anzeigen. Diese Teilnehmendeninterpretationen werden lokal (räumlich und zeitlich im Verlauf des Handelns), endogen (innerhalb der Handlungssituation) und - für die Analyse der multiplen Aktivitäten in Theaterproben besonders relevant - multimodal (durch Einbezug aller potentiell für eine Interaktion relevanten Ressourcen) in der Interaktion durch die Beteiligten erzeugt (Bergmann 1981: 12).

Auch wenn Stivers \& Rossano (2010: 53-54) herausstellen, dass bislang nach wie vor ungeklärt ist, was Menschen veranlasst, Handlungen als Accounts bestimmter Handlungen zu erkennen, zeigen ethnomethodologischen Studien von beispielsweise Unterrichtssituationen (Heap 1990) oder Gerichtssälen (Maynard \& Clayman 1991), dass die Accounts der Teilnehmenden die Merkmale der jeweiligen Settings konstituieren. Dabei gehen Beteiligte davon aus, dass andere Teilnehmende desselben Settings ein ähnliches Wissen in Bezug auf dieses haben (zum Beispiel durch Erfahrung, Tradition oder Ausbildung) und behandeln die Accounts innerhalb des Settings als dessen zentrale Eigenschaften (Garfinkel 1967: 8). Das bedeutet, dass Teilnehmende einer Situation einander ihr Verständnis einer Handlung innerhalb des aktuellen Kontextes anzeigen, was es anderen Interagierenden ermöglicht, auf dieses angezeigte Verständnis zu reagieren und ihrerseits die Interpretation der Situation anzuzeigen und so mitzukonstituieren. Damit wird das, was Teilnehmende einander öffentlich multimodal anzeigen, immer zur reflexiven Reaktion auf vorherige indexikalische Verweise in der situativen Interaktionshistorie. Versteht man Interaktionen als eine aufeinander bezogene Folge multimodal organisierter Interaktionsbeiträge, wird das gegenseitige Anzeigen (Displaying) der Teilnehmendeninterpretation der direkt vorausgegangenen Interaktionsbeiträge der anderen Teilnehmenden zur wichtigsten Informationsquelle für Interagierende sowie Interaktionsforschende (Mondada 2006: 127).

Das Display-Konzept im Zentrum der konversationsanalytischen Methodologie beschreibt also, dass „Sinn und Ordnung als solche erkennbar hergestellt werden und dass auch ihr Erkennen signalisiert wird“ (Deppermann 2000: 99).

15 Im Original: „visibly-rational-and-reportable-for-all-practical-purposes” (Garfinkel 1967: xii). 
Um sich gegenseitig ein Display ihres aktuellen Handelns (und damit auch ihre Interpretation vorausgegangener Interaktionsbeiträge) zur Verfügung zu stellen, können Interagierende auf zahlreiche Verfahren zurückgreifen, die nicht a priori auf bestimmte Modalitäten festgelegt sind. Vielmehr umfasst das Display-Konzept sowohl ein sprachliches Anzeigen einer Teilnehmendeninterpretation (etwa als Verstehensinferenz im Rahmen einer Antwort, vgl. Deppermann \& Helmer 2013) als auch multimodal organisierte embodied displays (Heath 1986; Mondada 2013).

Das next turn proof procedure fußt also darauf, dass Mitglieder eines Interaktionsensembles ihre Interaktionsbeiträge accountable herstellen, was es anderen Interagierenden ermöglicht daraufhin ihre Teilnehmendeninterpretation ebendieser Beiträge als multimodales Display anzuzeigen. An seine Grenzen stößt das auf Grundlage von Verbaldaten ${ }^{16}$ entwickelte Verfahren als Analysezugang hingegen immer dann, wenn keine sprachliche Orientierung in Bezug auf ein Phänomen erkennbar ist. Dies ist zum ersten immer dann der Fall, wenn nicht Handlungen rekonstruiert, sondern linguistische Einheiten betrachtet werden sollen. Besonders Arbeiten der Interaktionalen Linguistik müssen sich daher immer wieder damit auseinandersetzen, ob und was Teilnehmende in ihren Äußerungen einander anzeigen (Couper-Kuhlen \& Selting 2018: 24-26). Methodologische Vorschläge zur Modifikation des next turn proof procedures für interaktional-linguistische Arbeiten wie beispielsweise Kookkurrenz- (Wootton 1989) oder Rhythmusanalysen (Küttner 2014) setzen an diesem Punkt an.

Zum zweiten stellt die multimodale Verfasstheit von Interaktionen das next turn proof procedure immer dann auf die Probe, wenn sprachfreie Interaktionsbeiträge rekonstruiert werden sollen. Mit der multimodalen Perspektive rückt das Paradigma der körperlichen Anwesenheit als Voraussetzung für Interaktionssituationen in den Fokus. Denn Face-to-Face-Interaktionen sind immer multimodal. Diese Perspektive hat zur Folge, dass die klassischen Konzepte von Sprecher und Hörer noch weiter als sowieso schon in der Konversationsanalyse üblich zugunsten eines reflexiven Verständnisses von Interaktion zurücktreten. Wie bereits Goffman $(1963,1979)$ beschreibt, sind traditionell als Sender eingestufte Sprecher

16 Die verbalfokussierte Perspektive ist eher die Konsequenz aus dem frühen Forschungsinteresse der Gründer^innen, als eine programmatische Festschreibung. In Untersuchungen zu Eröffnungen in Telefongesprächen (z. B. Schegloff \& Sacks 1973), bei denen den Interagierenden ebenso wie den Forschenden nur auditiv übermittelte Signale zur Verfügung stehen, können Interaktionsprozesse rekonstruiert werden, ohne die audiovisuelle Komplexität einer multimodalen Situation mitanalysieren zu müssen. Dass außersprachliche Handlungen nichtsdestotrotz bereits in der frühen Konversationsanalyse mitgedacht werden, zeigen viele Beispiele in Harvey Sacks (1992: 569) Lectures, die sich mit dem RAUCHEN als sozialer und körperlich organisierter Aktivität befassen. 
ebenso Empfänger, wie Hörer auch Sender sind. Obwohl die sequentielle Ordnung der Interaktionsbeiträge außer Frage steht, bedeutet das, dass Teilnehmende einander konstant und unabhängig von ihrem Sprecherstatus multimodale Displays anbieten, auf die andere im Rahmen ihrer Teilnehmendeninterpretation reagieren (können). Grundlage dafür ist die gegenseitige Wahrnehmung innerhalb einer sozialen Situation (Goffman 1981: 84), in der zwei oder mehr Personen sich innerhalb seh- und hörbarer Reichweite voneinander befinden und diese Displays auditiv bzw. visuell verfolgen können (Monitoring). Ein an eine Person adressierter Interaktionsbeitrag beinhaltet daher ebenso ein körperliches Anzeigen des $^{\star}$ r Adressierten, inwiefern er*sie sich als Adressat*in versteht, wie auch die Reaktion des ${ }^{\star}$ Interagierenden auf dieses Display. Schaut der ${ }^{\star}$ die Adressierte zurück? Zieht er^sie die Augenbrauen zusammen? Nickt er`sie? All diese körperlichen Reaktionen des ${ }^{\star}$ r Adressierten wirken emergent auf die Produktion des Interaktionsbeitrags ein, noch bevor die Äußerung überhaupt abgeschossen ist (vgl. Goodwin 1979). Die Körper der Interagierenden fungieren in diesem Sinne als „semiotische Anzeigetafeln“ (Deppermann, Feilke \& Linke 2016: 4).

Diesem Ansatz folgen aktuell die meisten multimodal ${ }^{17}$ ausgerichteten konversationsanalytischen Arbeiten (z. B. Keevallik 2018; Mondada 2020; Goodwin 2021). Multimodal orientierte Konversationsanalytiker`innen greifen das für menschliche Interaktion zentrale Prinzip der Sequentialität auf und betrachten nicht nur Äußerungen, sondern alle Interaktionsbeiträge, die auf einen vorherigen oder nachfolgenden Beitrag Bezug nehmen. Aus dem next turn proof procedure wird dadurch ein „next-action proof procedure“ (Mondada 2016: 361), das alle multimodal organisierten Handlungen nach und während eines Interaktionsbeitrags dafür nutzt, lokale Teilnehmendeninterpretationen des laufenden Geschehens abzuleiten. Bei dieser Vorgehensweise ist häufig zu beobachten, dass die Analysen nach wie vor sprichwörtlich mit einem Fuß in der Verbalität stehen: Oft beinhaltet nämlich entweder der vorausgehende oder der nachfolgende Interaktionsbeitrag verbale Bestandteile, was das Erfassen von Teilnehmendeninterpretationen deutlich erleichtert. Vor einer Herausforderung stehen Interaktionsforschende immer dann, wenn es darum gehen soll Beteiligungsweisen von Aktivitäten zu betrachten, die nicht sprachlich organisiert sind (vgl. Hochuli 2019: 435). Will man bei der Analyse gleichzeitiger Relevanz multipler Aktivitäten nicht nur Aktivitäten mit sprachlicher Beteiligung berücksichtigen, wird also ein Verfahren benötigt, das neben der Verbalität auch andere Modalitäten des

17 In den frühen Arbeiten, die multimodale Ressourcen in die Konversationsanalyse integrieren (z. B. Goodwin 1980b, Goodwin 1984, Heath 1986, Schegloff 1984), wird der analytische Zugriff nicht explizit als ein multimodaler benannt, was den Eindruck bestärkt, dass die Konversationsanalyse eigentlich schon immer multimodal gedacht werden konnte. 
Körpers (und darüber hinaus) als gleichwertige Ressourcen mit eigenen internen Logiken in Interaktionssituationen analytisch fassen kann. Das bedeutet, dass die interaktive Ordnung (Goffman 1983) der Vollzugswirklichkeit (Garfinkel 1967) sowohl mithilfe einer schrittweisen Sequenzanalyse (Sacks, Schegloff \& Jefferson 1974) als auch als simultanes, multimodales Produkt des methodischen Accountable-machens und gegenseitigen Anzeigens der Teilnehmendeninterpretation (Displaying) rekonstruiert werden muss. Für dieses Vorhaben wird im Folgenden eine Erweiterung des next action turn procedures vorgeschlagen. Dabei soll nicht nur betrachtet werden, welche Displays durch die Teilnehmenden hergestellt werden, sondern es muss zwingend berücksichtigt werden, welche Beiträge die Interagierenden interaktiv verfolgen (Monitoring).

\subsection{Displaying und Monitoring als analytische Zugriffe bei verbaler Abstinenz}

Das Extrahieren der Teilnehmendeninterpretation verbal abstinenter Aktivitäten stellt, wie oben beschrieben, eine analytische Herausforderung in Konversationsanalysen mit konsequent multimodaler Perspektive dar, da „die für die Untersuchung verbalen Ausdrucks entwickelten Analyseverfahren und Konzepte nicht fraglos auf die anderen Modalitätsebenen übertragen“ werden können und in Bezug auf ihre „Übertragbarkeit und Adäquatheit methodisch reflektiert und empirisch beantwortet werden“ (Schmitt 2005: 19) müssen. Die zentrale Frage bleibt dabei: „How can we account for bodily activities?” (Deppermann 2013: 4). Eine Antwort auf diese Frage soll im Folgenden skizziert werden und dient so als Blaupause für den analytischen Zugriff auf verbal abstinente Aktivitäten im Rahmen dieser Studie.

Im Kern setzt die hier präsentierte Lösung für dieses methodische Problem am Prinzip des next turn/action proof procedure an. Anstatt jedoch auf die (sprachlichen) Reaktionen bezüglich vorausgegangener Turns zu schauen und davon die Teilnehmendeninterpretation abzuleiten, rücken nun alle Beteiligungsweisen bzw. Displays in den Fokus, die Interagierende füreinander öffentlich herstellen. Diese Displays sind multimodal organisiert und umfassen verbale Accounts (z. B. ein Okay) ebenso wie verbal abstinente Beteiligungsweisen (z. B. ein Nicken). Um allerdings zu verstehen, welche Handlungen Teilnehmende realisieren, reicht es nicht aus, sich lediglich die Reaktionen der Beteiligten auf bestimmte Interaktionsbeiträge anzuschauen. Es ist vielmehr notwendig nachzuvollziehen, welche dieser Beiträge sie interaktiv im Rahmen eines „display of availability“ (vgl. Heath 1986: 33) verfolgen. Damit wird das gegenseitige Monitoring der Interagierenden zur Voraussetzung für soziale Interaktion. Monitoring wird hier konsequent mul- 
timodal verstanden als alle Verfahren, auf die Interagierende zurückgreifen, um Displays anderer Beteiligter wahrzunehmen. Dies umfasst neben dem (visuellen) Beobachten auch das (akustische) Zuhören, das (haptische) Spüren sowie auch Riechen (und Schmecken).

Interagierende monitoren unter anderem die körperlichen Ressourcen, wie Gesten oder die Oberkörperorientierung, die ihnen Rückschlüsse auf das engagement display (Goodwin 1981) der anderen Beteiligten liefern. Mithilfe eines solchen engagement display als Orientierung eines ${ }^{\star} r$ Teilnehmenden auf eine ${ }^{\star} n$ anderen Teilnehmenden innerhalb einer sozialen Situation zeigen Interagierende einander an, welchen Teilnehmendenstatus sie einander zuschreiben. Interagierende mit aktivem engagement display machen sich so für andere Beteiligte kommunikativ verfügbar, während ein disengagement display Nichtverfügbarkeit anzeigt (Goodwin 1981:96). Das Display der Involvierung ${ }^{18}$ in eine Aktivität ist nach Goffman (1963) die Grundlage dafür, dass Beteiligte die jeweilige Aktivität als Teil einer sozialen Situation interpretieren können. Involvierung ist somit erkennbar als eine Leistung, mit der Teilnehmende den Grad der Verbundenheit zwischen sich und der jeweiligen Aktivität anzeigen:

INVOLVEMENT refers to the capacity of an individual to give, or withhold from giving, his concerted attention to some activity at hand - a solitary task, a conversation, a collaborative work effort. It implies a certain admitted closeness between the individual and the object of involvement, a certain overt engrossment on the part of the one who is involved. Involvement in an activity is taken to express the purpose or aim of the actor.

(Goffman 1963: 43-45)

Um zu entscheiden, ob und in welche Aktivität Ko-Interagierende involviert sind, monitoren Beteiligte nicht nur die körperlichen Ressourcen der Teilnehmenden sondern auch „semiotische Felder“ (Goodwin 2000a) außerhalb des Körpers. Dazu zählen unter anderem Bilder, Spielfelder und Tiefseediagramme (Goodwin 1995b) sowie Musik, Farben und Druckveränderung (Norris 2011) oder auch Vorgänge der Objektmanipulation (Nevile et al. 2014). Das verdeutlicht noch einmal, dass Multimodalität in Interaktionen keinesfalls mit Körperlichkeit gleichgesetzt werden sollte. Körperlich realisierte Praktiken in Interaktionen sind in der Regel multimodal, aber nicht jede multimodal organisierte Interaktion benötigt zwingend eine körperliche Handlung (siehe für empirische Beispiele dazu Krug angenommen).

18 Goffman (1963: 43-45) unterscheidet bei der Involvierung zwischen Hauptinvolvierung (,main involvement“), die den Großteil der Aufmerksamkeit eines Menschen in Anspruch nimmt und Nebeninvolvierung (,side involvement”), die ausgeführt werden kann, ohne die Aktivität mit Hauptinvolvierung zu stören, z. B. das SummEN während des ARBEITENS. Da in dieser Studie Aktivitäten in Abhängigkeit ihrer intersubjektiven Gestaltung in Einzel- und Ensembleaktivitäten unterschieden werden, wird die Goffman'sche Differenzierung im weiteren Verlauf nicht verfolgt. 
Das Monitoring multimodal organisierter Displays in Face-to-Face-Interaktionen stellt einerseits eine Grundvoraussetzung für das Gelingen von kommunikativen Prozessen dar (Goodwin \& Goodwin 1996: 88). Andererseits handelt es sich beim Monitoring auch um eine interaktive Beteiligungsweise an der jeweiligen Aktivität (vgl. Müller \& Bohle 2007: 136). Indem also die Teilnehmenden füreinander multimodale Displays herstellen und diese monitoren, wird ein analytisches Zugriffsverfahren möglich, das nach der Logik des next turn/action proof procedure die Teilnehmendeninterpretation aus der Reaktion auf das vorher gemonitorte Display gewinnen kann. Von essentieller Bedeutung bei diesem analytischen Zugang ist, die multimodalen Ressourcen nicht isoliert zu betrachten, sondern als Teil einer multimodalen Gestalt zu begreifen. Denn es ist nicht eine einzelne Geste, die ein Display einer spezifischen Handlung herstellt. Vielmehr bildet das Zusammenwirken von Körperorientierung, Blickkontakt, Sprache und möglicherweise auch Mimik als multimodales Paket ein Display, das im situativen Kontext der gerade sich im Vollzug befindlichen Aktivität spezifische Handlungen darin erkennbar werden lässt. Das bedeutet, dass Displays immer als multimodale Gestalten behandelt werden, wenn die Teilnehmenden sich in Hörund Sehweite (mit den „naked senses [...] in one another's immediate presence“, Goffman 1964: 135) befinden und wechselseitiges Monitoring-Verhalten (,mutual monitoring“, Goodwin 1980b) zeigen. Obwohl die Wahrnehmung nicht mit dem Sehen gleichzusetzen ist (Merleau-Ponty 2016 [1946]), kommt der Ressource Blick hier eine besondere Rolle $^{19} \mathrm{zu}$ :

First, sight begins to take on an added and special role. Each individual can see that he is being experienced in some way, and he will guide at least some of his conduct according to the perceived identity and initial response of his audience.

(Goffman 1963: 16)

Eine solche über wechselseitiges Monitoring hergestellte „Wirbeziehung“ (Schütz 1932: 181) zeigt den Teilnehmenden (und damit auch den Forschenden) an, dass die sich monitorenden Interagierenden als kopräsente Beteiligte verstehen (Goodwin 1981; Goffman 2001; Clark \& Krych 2004). Die mithilfe des wechselseitigen Monitorings hergestellte Wahrnehmungswahrnehmung (Hausendorf 2009) erzeugt somit einen interaktionalen Rahmen, in dem Teilnehmende dem Display der anderen einen Sinn zuschreiben können und ihre Interpretation dieser Zuschreibung als Teilnehmendeninterpretationen anzeigen können. Unter dieser Voraussetzung lassen sich auch Beteiligungsweisen an Aktivitäten ohne

19 Dieser besonderen Rolle visueller Beteiligungsweisen bei der Koordination multipler Aktivitäten wird besonders in Kapitel 7 Rechnung getragen. Dort wird mithilfe mobiler Eye-TrackingBrillen Monitoring in Situationen mit Multiaktivitäten gemessen und in Relation zu koordinativen Verfahren bei der Bearbeitung von Gleichzeitigkeit in Interaktionen analysiert. 
verbale Komponente als aufeinander bezogen konversationsanalytisch rekonstruieren. Dies ist im Rahmen der vorliegenden Fragestellung insofern wichtig, als in dieser Studie untersucht wird, wie Interagierende die gleichzeitige Relevanz häufig sprachfreier Einzel- mit oftmals sprachintensiven Ensembleaktivitäten in Hinblick auf die strukturelle (In)Kompatibilität der dafür verwendeten Beteiligungsweisen koordinieren. 